\title{
A Survey of Communications and Networking Technologies for Energy Management in Buildings and Home Automation
}

\author{
Aravind Kailas, Valentina Cecchi, and Arindam Mukherjee \\ Department of Electrical and Computer Engineering, University of North Carolina Charlotte, Charlotte, NC 28223-0001, USA \\ Correspondence should be addressed to Aravind Kailas, aravind.kailas@uncc.edu
}

Received 11 June 2011; Accepted 5 December 2011

Academic Editor: Tin-Yu Wu

Copyright () 2012 Aravind Kailas et al. This is an open access article distributed under the Creative Commons Attribution License, which permits unrestricted use, distribution, and reproduction in any medium, provided the original work is properly cited.

With the exploding power consumption in private households and increasing environmental and regulatory restraints, the need to improve the overall efficiency of electrical networks has never been greater. That being said, the most efficient way to minimize the power consumption is by voluntary mitigation of home electric energy consumption, based on energy-awareness and automatic or manual reduction of standby power of idling home appliances. Deploying bi-directional smart meters and home energy management (HEM) agents that provision real-time usage monitoring and remote control, will enable HEM in "smart households." Furthermore, the traditionally inelastic demand curve has began to change, and these emerging HEM technologies enable consumers (industrial to residential) to respond to the energy market behavior to reduce their consumption at peak prices, to supply reserves on a as-needed basis, and to reduce demand on the electric grid. Because the development of smart gridrelated activities has resulted in an increased interest in demand response (DR) and demand side management (DSM) programs, this paper presents some popular DR and DSM initiatives that include planning, implementation and evaluation techniques for reducing energy consumption and peak electricity demand. The paper then focuses on reviewing and distinguishing the various state-of-the-art HEM control and networking technologies, and outlines directions for promoting the shift towards a society with low energy demand and low greenhouse gas emissions. The paper also surveys the existing software and hardware tools, platforms, and test beds for evaluating the performance of the information and communications technologies that are at the core of future smart grids. It is envisioned that this paper will inspire future research and design efforts in developing standardized and userfriendly smart energy monitoring systems that are suitable for wide scale deployment in homes.

\section{Introduction}

Residential energy consumption and the amount of pollution emitted from the electric generators create side effects that are not beneficial to public health and well-being, including increased pollution in the air and water $\left(\mathrm{CO}_{2}\right.$ and other greenhouse gases, mercury, and other trace elements and particulate matter), and the depletion of finite resources [1]. "Green Smart Home Technologies" are aimed at reducing the footprint of greenhouse gases by efficient energy management in residential buildings. Studies have shown that the display of real-time information on consumption can result in reductions of up to $30 \%$ by enabling end users to consume responsibly and manage effectively [2]. In recent times, more so than ever, the consumer has become more "green" conscious and therefore is looking for realtime visibility of energy consumption [3]. Further, the market for residential energy management is poised to grow dramatically due to increased consumer demand and new government and industry initiatives [4]. Smart homes have been studied since 1990s, and their primary focus has been resident comfort [5]. They employ energy efficiency by occupancy check or adaptability to outside conditions. However, they are not automatically a component of the smart grid. Their integration to smart grid is an active topic [6-8]. With this in mind, this paper motivates future research in the area of home area networking by revisiting the concepts of smart grids and smart homes and summarizing the state of the art in home energy management (HEM) communications and control technologies.

1.1. Bringing Smart Grids to Green Smart Homes. Smart grid is accelerating the energy value change transformation, 
and will enable electricity distribution systems to manage alternative energy sources (e.g., solar and wind), improve reliability, facilitate faster response rates to outages, and manage peak-load demands. Building a smart digital meter, the advanced metering infrastructure (AMI) is a first step and would enable processing and reporting usage data to providers and households via two-way communication with the utility offices [9-11]. In recent years, there have been a lot of initiatives on the part of the government, utilities companies, and technology groups (e.g., standards committees, industries, alliances, etc.) for realizing smart grids for green smart homes [12]. Government initiatives include mandating upgrades to the grid and adding intelligence to meters that measure water, gas, and heat. The market for smart home products, such as lighting and HVAC controls, in-home utility monitors, and home security systems, is also on the rise, driven in part by the desire to conserve energy and by the expansion of home automation services and standards-based wireless technologies. Further, energy directives and smart grid initiatives have attracted hundreds of companies with energy management systems including General Electric, Cisco, Google, and Microsoft. Efforts are underway to design new standards, protocols, and optimization methods that efficiently utilize supply resources (i.e., conventional generation, renewable resources, and storage systems) to minimize costs in real time. In other words, smart grid technologies so far focus on integrating the renewable energy resources to the grid to reduce the cost of power generation and integrating these resources requires storage systems. Smart grids can be potent tools in helping consumers reduce their energy costs, but consumers have several concerns that could inhibit rapid adoption. In order to maximize smart grids, utilities and suppliers of energy management solutions must first educate consumers about the benefits of these advanced systems and then package these solutions so that capabilities and advantages are obvious to consumers and easily integrated into their lifestyles.

\subsection{Home Energy Management and Home Area Networks.} The term home area networks (HANs) has been used loosely to describe all the intelligence and activity that occurs in HEM systems, and this section describes the concepts of HEM systems and HANs. Stated simply, HANs are extensions of the smart grid and communications frameworks, much like the familiar local area networks (LANs), but within a home [10]. Instead of a network of servers, printers, copiers, and computers, the HAN connects devices that are capable of sending and receiving signals from a meter and/or HEMS applications. Wired or wireless, there are tradeoffs that involve power consumption, signaling distance, sensitivity to interference, and security. The main point here is that HANs are not energy management applications, but enable energy management applications to monitor and control the devices on the home network.

With limited data input and display capabilities, inhome displays (IHDs) function as a visual indicator of the electricity rates at any point in time. Moreover, IHDs

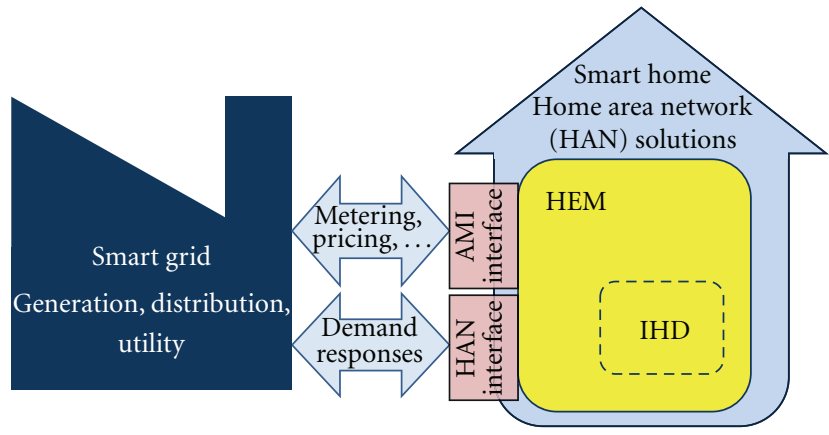

FIgURE 1: Realizing smart grids in smart homes.

are one-way communication devices, meaning the user can only monitor, but not take, real-time actions and provide feedback to the HAN like the HEM systems. So, HANs and IHDs still need an energy management application, an HEM solution $[13,14]$, in order to gain the most benefit from these smart grid components. A web-based portal for an HEM system is the best interface to the utility billing and demand response programs, because it enables the easiest execution and control of intelligent appliances that can be "enrolled" into such programs. A HEM solution would enable the user to recall the optimized presets for sustainable energy-saving, get suggestions on energy efficiency improvements, and see how ones' energy management compares to others in ones' peer group or neighborhood [10]. A basic representation of a smart grid-smart home interface that uses a variety of different networking topologies across the different domains and subdomains is illustrated in Figure 1, and the focus of this paper is HEM systems and the HAN technologies.

\subsection{Benefits of HEM}

(1) Minimize Energy Wastage. Home automation and realtime energy monitoring makes energy savings feasible. For example, lighting control is not about reducing light, but facilitating the correct light when and where required, while reducing wastage. Energy savings can also be realized according to occupancy, light level, time of day, temperature, and demand levels, for example, opening and closing blinds and shutters automatically, based on the time of day and amount of light to optimize the mix of natural light and artificial light or according to the temperature difference between indoor and outdoor to optimize heating, ventilating, and air conditioning (HVAC) power consumption. Home automation also minimizes energy wastage without affecting usage with occupancy detection, and planning appliance control and varies settings depending on demand response levels. Energy savings come off the highest rate, and HEMs help in monitoring the usage to track the highest electricity rate one is paying. For example, consider a 100 Watts light bulb (in the state of California, USA), a common electrical device in homes. Leaving the light on all month would cost $\$ 7.92$ if the overall energy usage is very low, but increases to $\$ 25.20$ for substantially higher overall usage [2]. 
So, keeping that 100 Watt light off would save the household $\$ 25.20$ per month [2].

(2) Peace of Mind. Home energy management is important because it provisions time scheduling and predictive scheduling that ensures peace of mind while yielding energy savings. With preset scheduling, the user does not need to turn them on all the time and thus minimize energy consumption. Further, lights and TV turned on will help to discourage potential intruders while you are away from home. Safety locks and security systems can be enabled as well; lighting and sound/motion sensors can be connected to the HEM that track activity 24 hours a day and alerts the user and the local police or fire department if and when needed.

(3) Eco-Friendly. As climate change becomes an increasingly real concern, energy efficiency has become top priority in homes and businesses alike. When describing a green home, energy efficiency refers to every aspect of energy consumption, from the source of electricity to the style of light bulbs. Reducing energy consumption requires a longterm behavioral change, the first step being an investigation of the current carbon footprint of residential and office buildings. HEMs aid in this change by helping the user monitor the usage (e.g., heat, light, and power in homes) and by offering suggestions on how to cut down $\mathrm{CO}_{2}$ emissions, a primary cause of global climate change. Continuing with the same example from [2], and using the "Terra Pass Carbon Footprint Calculator," a reduction in the $\mathrm{CO}_{2}$ emissions (from the household) by a factor of more than $60 \%$ would be possible.

(4) Well-Being of Residents. With the average family spending a huge amount annually on gas and electricity supplies alone, it certainly makes sense to do everything possible to reduce household utility bills. Good energy management within the home brings about this reduction, thereby increasing available capital. HEM systems also increase the transparency and improve the billing service. Such systems make life easy by providing the user with control and management, which will help manage ones time better and thus help to reduce stress. Reducing the energy consumption in a household by about $23 \%$ cut the monthly bills by over a third [2].

(5) Public Good. In terms of public good, four things can occur simultaneously when homes are energy efficient: (i) finite energy supplies are not depleted as quickly, (ii) emissions are reduced (including all the corresponding benefits associated with reduced emissions), (iii) consumers save money, and (iv) consumers increase net disposable income. With low-to-moderate income residents, saving money on utilities and spending those savings elsewhere can be a significant quality of life factor. An additional public benefit can result from energy-efficient housing. When government agencies serve as the housing provider for low-income residents, energy efficiency can contribute to taxpayer savings. Money can be saved when the government does not have to finance wasteful energy practices with public housing. An example of a governmental agency collaboration designed to reduce energy use in public housing is the partnership between local housing agencies (LHAs) (agencies who receive program funding from the department of housing and urban development (HUD)) and the DOE Rebuild America program.

In summary, HEM systems are a step more advanced than previous energy-saving appliances that provides even more eco-friendly performance through the use of sensor technology. HEM systems allow energy monitoring, automation of appliances, and control system settings to respond to demand response levels. Thus, planning advance personal energy consumption plans is encouraged to leverage from rebates/incentives for green homes and to benchmark oneself on a community level. To the best knowledge of the authors, this is the first comprehensive tutorial on the state of the art in home area communications and networking technologies for energy and power management. This paper also presents a classification of the many affordable smart energy products offered by different companies that are available in the market.

\section{Background on Demand Response (DR) and Demand Side Management (DSM) Programs}

In support of "smart grid" initiatives, several emerging technologies and techniques have been presented in the past decade. These techniques include, among others, advanced metering infrastructure (AMI) and two-way communication, integration of home area network (HAN) and home automation, and a push to invest in renewable microgeneration. The traditionally inelastic demand curve has began to change, as these technologies enable consumers (industrial to residential) to respond to the energy market behavior, reducing their consumption at peak prices, supplying reserves on a as-needed basis, and reducing demand on the electric grid [15]. Therefore, the development of smart grid-related activities has resulted in an increased interest in demand response and demand side management programs.

Demand response programs are used to manage and alter electricity consumption based on the supply, for example, during a reliability event (Emergency DR), or based on market price (Economic DR) (e.g., [16, 17]). These programs can involve curtailing electric load, as well as utilizing local microgeneration (customer owned Distributed Generation). DR programs can be incentive-based programs (IBPs), classical and market-based, or priced based-programs (PBP) [17].

Demand side management (DSM) refers to planning, implementation, and evaluation techniques, including policies and measures, which are designed to either encourage or mandate customers to modify their electricity consumption, in terms of timing patterns of energy usage as well as level of demand. The main objective is to reduce energy consumption and peak electricity demand.

DR and DSM initiatives can benefit customers, utilities, as well as society as a whole. From the customer perspective, 
these programs can help reduce the electric bill and is possibly incentivized by the utility (e.g., through tax credits). From a utility perspective, in addition to reducing supply costs (generation, transmission, and distribution), benefits also include deferral of capital expenditure on increasing system capacity, improved system operating efficiency and reliability, and better/more data to be used for planning and load forecasting. Society as a whole benefits also through the reduction of greenhouse gas emissions, due to the decrease (or nonincrease) in energy consumption and peak demand and the avoided expansion of grid generation capacity. Major benefits of DSM are summarized in Table 1.

As part of DSM initiatives, several objectives are included, mainly load management and energy efficiency [18] (refer to Figure 2). Under the load management objectives, we have peak clipping, valley filling, and load shifting. Energy efficiency, or conservation, involves a reduction in overall electricity usage. Electrification and flexible load shape, also shown in Figure 2, involve, respectively, programs for customer retention and development of new markets and programs that utilitie setup to modify consumption on an as-needed basis (i.e., customers in these programs will be treated as curtailable loads). DSM concepts have been studied since the 1980s and early 1990s; reports and survey on the subject were published by the Electric Power Research Institute (EPRI) and the North American Electric Reliability Corporation (NERC) [19-21], among others.

In the past decade, the focus on smart grid applications and progress in communication protocols and technologies has improved the communication ability between electricity suppliers and end-use consumers, which would allow active deployment of DR at all times (demand dispatch [22]), not just event-based DR. Customers are then able to monitor and control their load in real time and to possibly trade in the energy market. This requires the use of sophisticated energy management system (EMS) to control equipment and appliances [23].

In [24], an optimized operational scheme for household appliances is introduced through the use of a demandside management- (DSM-) based simulation tool. The tool uses a particle swarm optimization algorithm to minimize customers cost and determine a source management technique. In the 1989 paper [25], the authors describe a system used to control electricity usage in homes or small businesses, by shifting some of the load from the peak to the valley and using a real-time variable pricing scheme. The proposed system uses a telephone to power line carrier (PLC) interface, a meter that measures energy with variable pricing, and a controller that adjusts energy utilization based on price. In [26], the authors developed, using mixed integer linear programming, a home energy management system (HEMS), which provides optimum scheduling for operation of electric appliances and controls the amount of power provided back to the grid from the excess local photovoltaic generation. In [27], the authors present a common service architecture developed to allow end-users interaction with other consumers and suppliers in an integrated energy management system. The architecture would facilitate users with renewable micro-generation to integrate with the electric grid, through the use of a central coordinator inside their home gateway. In [28], a multiscale optimization technique for demandside management is presented. A home automation system is proposed, which dynamically takes into account user comfort level as well as limits on power consumption. In [29], a novel strategy for control of appliances is proposed and utilizes a home automation and communication network. The goal of the proposed technique is to provide continued service, at possibly reduced power consumption levels, during power shortages. In [30], communication methodologies amongst control devices in home automation systems are demonstrated. Specifically, communication over a power line is presented to enable control of appliances in building/home energy management systems. In [31], a home automation system which controls household energy consumption is proposed. The system takes into account predicted/anticipated events and uses a tabu search to maximize user comfort and minimize consumption costs. In [32], control mechanisms to optimize electricity consumption within a home and across multiple homes in a neighborhood are presented and evaluated. Energy management controllers (EMCs) are assumed to control appliances operation based on energy prices and consumers preset preferences. The authors first show that a simple optimization model used for determining appliance time of operation purely based on energy price may actually result in higher peak demand. An EMC optimization model, based on dynamic programming, which also accounts for electricity capacity constraints, is then presented. A distributed scheduling mechanism is also proposed to reduce peak demand within a neighborhood.

To summarize, DSM refers to planning, implementation, and evaluation techniques, including policies and measures, which are designed to either encourage or mandate customers to modify their electricity consumption, in terms of timing patterns of energy usage as well as level of demand. The main objective is to reduce energy consumption and peak electricity demand. Potential research in this area should focus on identifying optimized system level hardware-software codesigned solutions to implement the DSM functionalities in the most energy-efficient manner to respond to the dynamically changing operating environment of the HAN under real-time constraints.

\section{HAN Communications and Network Technologies}

The energy management system is at the heart of green buildings and enables home energy control and monitoring, providing benefits to both consumers and utilities. The HEM system intelligently monitors and adjusts energy usage by interfacing with smart meters, intelligent devices, appliances, and smart plugs, thereby providing effective energy and peak load management. The platform for this communication is the HAN, and this section reviews the communications and network technologies for HAN for interworking the HEM to end points and smart meters [33]. The cost associated with HEM applications, as can be seen from Figure 3, is significantly lower compared to other home applications because 
TABLE 1: DSM Benefits.

\begin{tabular}{lcc}
\hline Customer benefits & Utility benefits & Societal benefits \\
\hline Satisfy demand for electricity & Lower cost of service & Conserve resources \\
Reduce costs & Improve efficiency and flexibility & Reduce environmental impact \\
Improve service & Reduce capital needs & Protect environment \\
Improve lifestyle and productivity & Improve customer service & Maximize customer welfare \\
\hline
\end{tabular}

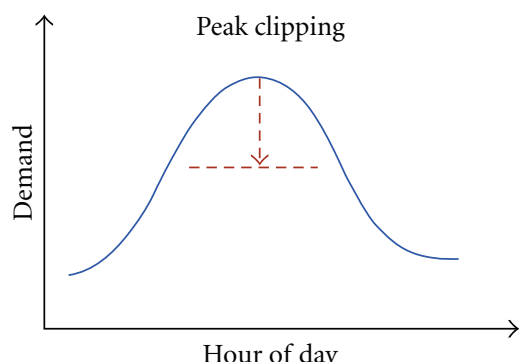

(a)

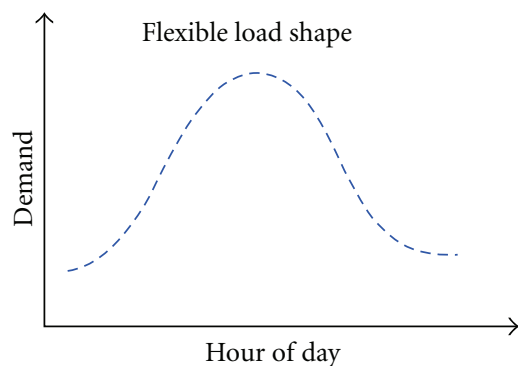

(d)

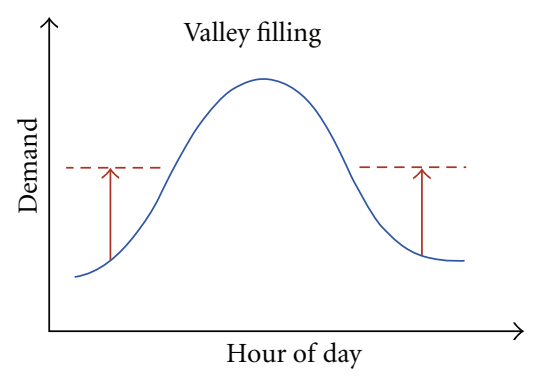

(b)

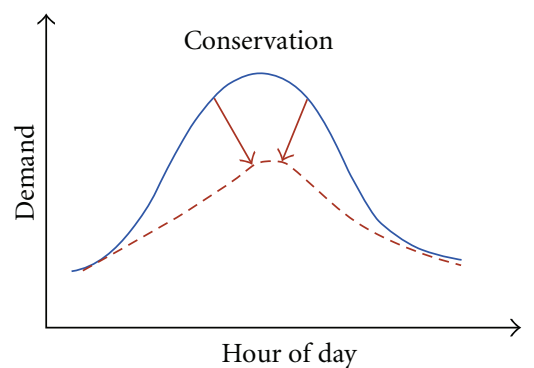

(e)

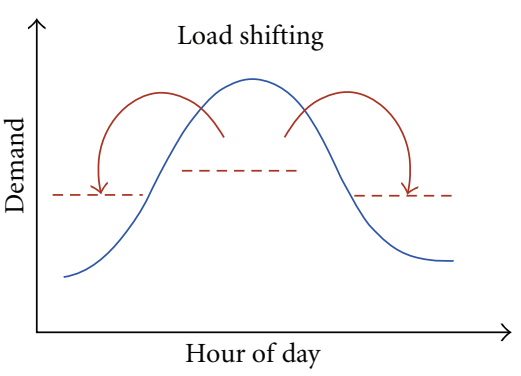

(c)

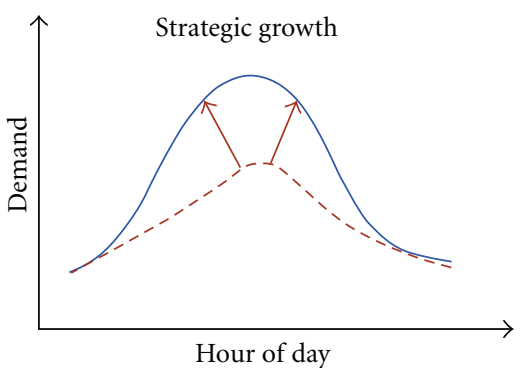

(f)

Figure 2: DSM-load shape objectives.

of the differing functionalities. For example, HANs comprise command-based systems that require very short acquisition time for sending data to multiple destinations, and this cuts down the data rate and the bandwidth requirements compared to link-based systems (e.g., communication and entertainment systems) that need a reliable point-to-point communication link for longer periods of time.

Internet protocol (IP) is a protocol used for communicating data within a packet-switched internetwork and is responsible for delivering data from source to destination based on an IP address. Being the foundation on which the Internet is built and communication is achieved, IP is a single layer within a multilayer suite known as the TCP/IP stack. Due to this abstraction, IP can be used across a number of different heterogeneous network technologies. Due to the ease of interoperability, ubiquitous nature, widespread adoption, and work being performed to create a lightweight interface, IP is being seen as essential to the success of HAN and smart grid development. As the significance of devices communicating within the HAN increases, so does the requirement for usable IP addresses. Very broadly, the different technologies (comprising specifications for the physical and network layers) can be classified based on the transmission medium into wired and wireless, as is shown in Figure 4 .

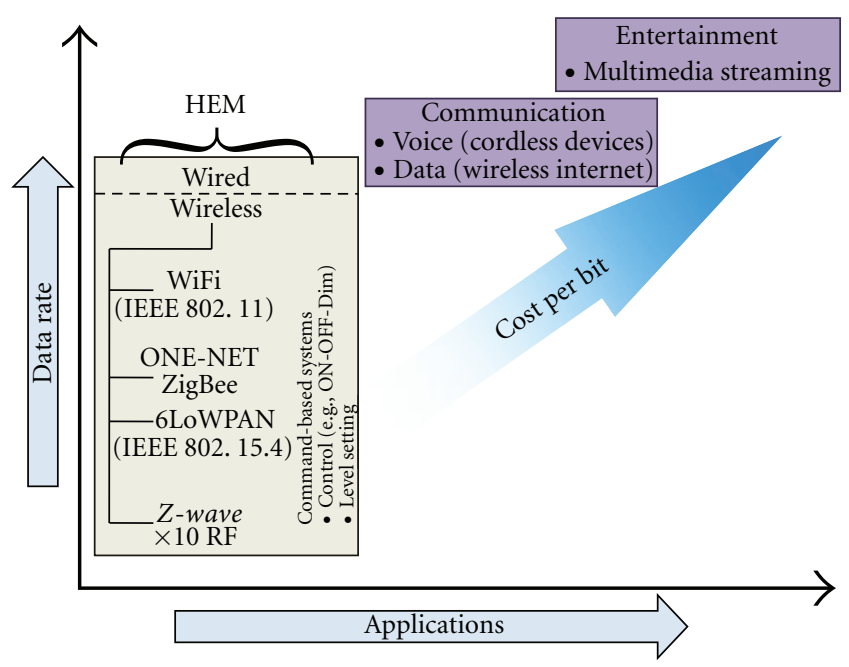

Figure 3: The cost and use of wired and wireless technologies for different home applications.

3.1. Wired HANs. First, we discuss the technologies in which the transmission mediums are electronic wiring, telephone lines, coaxial cables, unshielded twisted pairs, and/or optical fibers. HomePlug, a power line communication technology 


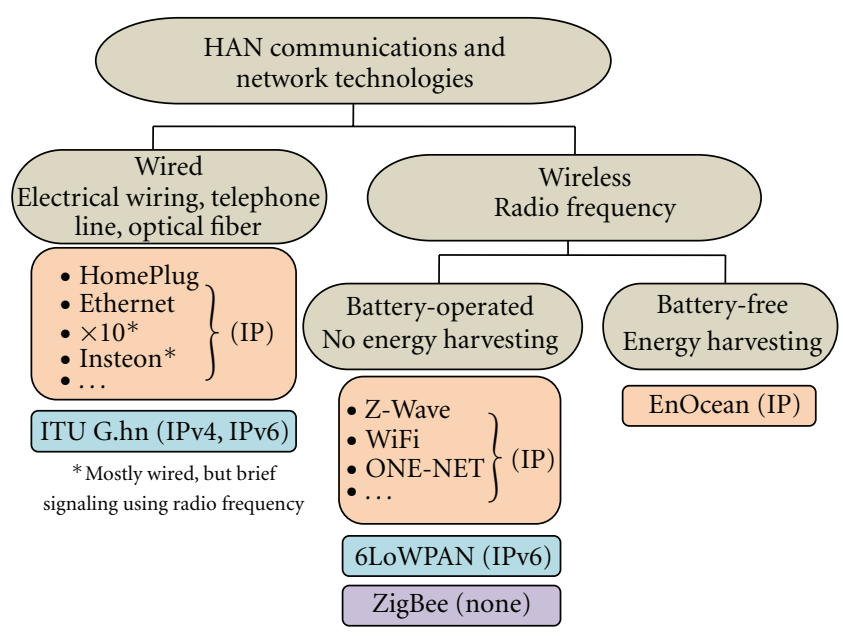

FIGURE 4: Communications and networking possibilities for a home area network.

that uses the existing home electricity wiring to communicate, is widely adopted for high-speed wired communication applications (e.g., high-quality, multistream entertainment networking) with a mature set of standards. Ethernet is a very common technology and supports a range of data rates using either unshielded twisted pairs (10 Mbps-1 Gbps) or optical fibers (as high as $10 \mathrm{Gbps}$ ). It utilizes a common interface found in a number of household equipment, including computer, laptops, servers, printers, audio video (AV) equipments, media, and game consoles. Ethernet may not be appropriate for connecting all devices in the HAN (especially appliances) due to the high cost and power requirements plus the need for separate cabling back to a central point.

$X 10$ is a technology (and an international and open industry standard) that uses power line wiring for signaling and control of home devices, where the signals involve brief radio frequency bursts representing digital information. However, it suffers from some issues such as incompatibility with installed wiring and appliances, interference, slow speeds, and lack of encryption. Insteon addresses these limitations while preserving the backward compatibility with $\mathrm{X} 10$ and enables the networking of simple devices such as light switches using the powerline (and/or radio frequency $(\mathrm{RF})$ ). All Insteon devices are peers, meaning each device can transmit, receive, and repeat any message of the Insteon protocol, without requiring a master controller or routing software. All the previously described technologies support popular protocols like the IP and hence can easily be integrated with IP-based smart grids. More recently, ITU G.hn has been developed by the International Telecommunication Union (ITU) and promoted by HomeGrid Forum. It supports networking over power lines, phone lines and coaxial cables, and the expected data rates up to 1 Gbps. ITU G.hn provides secure connections between devices supporting IP, IPv4, and IPv6 and offers advantages such as the ability to connect to any room regardless of wiring type, self-installation by the consumer, built-in diagnostic information, and selfmanagement as well as multiple equipment suppliers.
3.2. Wireless HANs. Next, we discuss wireless networking of low-cost, low-power (battery-operated) control networks for applications such as home automation, security and monitoring, device control, and sensor networks. The low-cost ZigBee-based solutions allow wide deployment in wireless control and monitoring applications; the low power usage allows longer life with smaller batteries (up to 10 years) and the mesh networking provides high reliability and broader range.

Z-Wave, a proprietary wireless communications technology designed specifically to remote control applications in residential and light commercial environments [34], is popular because of the following reasons. Unlike WiFi and other IEEE 802.11-based wireless LAN systems that are designed primarily for high-bandwidth data flow, the ZWave radio frequency (RF) system operates in the sub Gigahertz frequency range $(\approx 900 \mathrm{MHz})$ and is optimized for low-overhead commands such as ON-OFF-DIM (as in a light switch or an appliance), raise-lower (as in a volume control), and Cool-Warm-Temp (as in a HVAC) with the ability to include device metadata in the communications. As a result of its low power consumption and low cost of manufacture, Z-Wave is easily embedded in consumer electronics products, including battery-operated devices such as remote controls, smoke alarms, and security sensors. More importantly, Z-Wave devices can also be monitored and controlled from outside of the home by way of a gateway that combines Z-Wave with broadband Internet access.

WiFi is a popular IP-based wireless technology used in home networks, mobile phones, video games, and other electronic devices. Support is wide spread with nearly every modern personal computer, laptop, game console, and peripheral device provides a means to wirelessly access the network via WiFi. Another IP-based wireless technology is the ONE-NET, also an open-source standard that is not tied to any proprietary hardware or software and can be deployed using a variety of low-cost off-the-shelf radio transceivers and microcontrollers from various manufacturers.

6LoWPAN (also a standard from the Internet Engineering Task Force (IETF)) optimizes IPv6, the next-generation IP communication protocol for internetworks and the Internet [35], for use with low-power communication technologies such as the IEEE 802.15.4-based radios [36], enabling transfer of small packet sizes using low bandwidth. It is primarily aimed at evolving the current IPv4 protocol which is predicted to be exhausted of address space in 2011. Operation of the 6LoWPAN involves compressing 60 bytes of headers down to just 7 bytes. The target for IP networking for low-power radio communication is the applications that need wireless internet connectivity at lower data rates for devices with very limited form factor. 6LoWPAN allows communication with devices across the Internet without having to go through ZigBee-to-IP translation.

Finally, EnOcean technology efficiently exploits applied slight mechanical excitation and other potentials from the ambiance (motion, pressure, light, and temperature) using the principles of energy harvesting for networking selfpowered wireless sensors, actuators, and transmitters. In order to transform such energy fluctuations into usable 
TABLE 2: Summary of communications and networking technologies for home area networks.

\begin{tabular}{|c|c|c|c|c|}
\hline Connectivity & Technology & Max speed per channel & Range & Adoption rate \\
\hline \multirow{5}{*}{ Wired } & HomePlug (IEEE P1901) & $14-200 \mathrm{Mbps}$ & $300 \mathrm{~m}$ & Medium \\
\hline & Ethernet (IEEE 802.3) & $10-1000 \mathrm{Mbps}$ & $100 \mathrm{~m}$ & Extremely high \\
\hline & X10 (X10 standard) & $50-60 \mathrm{kbps}$ & $300 \mathrm{~m}$ & Medium \\
\hline & Insteon (X10 standard) & $1.2 \mathrm{kbps}$ & $3000 \mathrm{~m}$ & Medium \\
\hline & ITU G.hn (G.hn) & Up to $1 \mathrm{Gbps}$ & - & Not widely \\
\hline \multirow{8}{*}{ Wireless } & Z-Wave (Zensys, IEEE 802.15.4) & $40 \mathrm{kbps}$ & $30 \mathrm{~m}$ & Widely \\
\hline & WiFi (IEEE 802.11, IEEE 802.15.4) & 11-300 Mbps & $100 \mathrm{~m}$ & Extremely high \\
\hline & ONE-NET (Open-source) & $38.4-230 \mathrm{kbps}$ & $\begin{array}{l}500 \mathrm{~m} \text { (outdoors) } \\
60-70 \mathrm{~m} \text { (indoors) }\end{array}$ & Not widely \\
\hline & \multirow{3}{*}{ 6LowPAN (IEEE 802.15.4) } & $250 \mathrm{kbps}(2.4 \mathrm{GHz})$ & \multirow{3}{*}{$10-75 \mathrm{~m}$} & \multirow{3}{*}{ Medium } \\
\hline & & $40 \mathrm{kbps}(915 \mathrm{MHz})$ & & \\
\hline & & $20 \mathrm{kbps}(868 \mathrm{MHz})$ & & \\
\hline & ZigBee (IEEE 802.15.4) & $\begin{array}{l}250 \mathrm{kbps}(2.4 \mathrm{GHz}) \\
40 \mathrm{kbps}(915 \mathrm{MHz})\end{array}$ & $10-75 \mathrm{~m}$ & Widely \\
\hline & EnOcean (EnOcean standard) & $120 \mathrm{kbps}$ & $30 \mathrm{~m}$ & Not widely \\
\hline
\end{tabular}

electrical energy, electromagnetic, piezogenerators, solar cells, thermocouples, and other energy converters are used. The transmission range is around $30 \mathrm{~m}$ inside the building, and this technology allows for wireless gateway connectivity with common automation systems. The wired and wireless communications described thus far have been summarized in Table 2.

\section{HEM Hardware}

The imminent penetration of HEM systems in green homes has created a new market segment (HEM) for embedded hardware providers. In June 2010, Cisco System unveiled its home energy controller (HEC), which is part of a much larger smart grid infrastructure that spans solutions for utilities, substation networks, smart meter networks, and the home network. The HEC has a 7-inch user interface Tablet that runs Ubuntu Linux, powered by a $1.1 \mathrm{GHz}$ Intel Atom processor. Supplementing the HEC on the utility side is Ciscos Home Energy Management Solution, which gives utility companies the right tools to enhance customer satisfaction and effectively implement demand management, load shedding, and pricing programs for residential deployments. Figure 5 shows Ciscos HEC architecture.

Using the HEC, consumers can take advantage of special energy pricing programs, demand response can be managed, and electric vehicle integration becomes a reality. The HEC provides (1) the user engaging and easy-to-use energy management applications to monitor and budget energy use and control thermostats and appliances, (2) the utility of the ability to provision and manage a home area network (HAN) that monitors and controls energy loads, and (3) highly secure end-to-end data communications across wired and wireless media and networking protocols. The HEC is a networking device that coordinates with the networks in the home and the associated security protocols, such as ZigBee (communication with smart appliances),
WiFi (communication with the home network), and PLC and ERT (communications with utilities). To monitor and control energy loads such as heating, ventilating, and air conditioning (HVAC) systems, pool pump, water heaters, TVs, computers, and other devices, consumers will need to wirelessly connect the appropriate compatible, tested peripherals to the HEC. Cisco is currently in trials with utilities for the home energy controller.

To scale and support devices implemented in residential deployments, Ciscos Energy Management Software is deployed in utility facilities, and its hosted services help utilities provide personalization and data to increase customer satisfaction for energy programs. These services include

(i) provisioning and management capabilities,

(ii) unique, customized look and feel for devices,

(iii) mass firmware updates to thousands of devices,

(iv) integration with utility back-end applications and third party software.

During the last quarter of 2010, both Freescale Semiconductor and Intel Corp. have announced reference designs targeting the HEM market. Freescale demonstrated its Home Energy Gateway (HEG) reference platform in September 2010 in Europe. Freescale's, Home Energy Gateway reference platform is based on the i.MX ARM9 SoC that is both flexible and scalable and based on ZigBee Smart Energy 1.0 mesh architecture for bidirectional control Figure 6. The HEGs controller integration allows for a low bill-of-materials cost. Freescale's HEG includes a central hub that links smart meters, smart appliances, and smart devices in the home area network (HAN) and collects and reports power usage data. The Freescale HEG allows every point of the smart home to be connected and controlled from a central point, enabling power efficiency and energy optimization. The HEG links to a WAN for remote control and monitoring by the utility and communications service provider. 


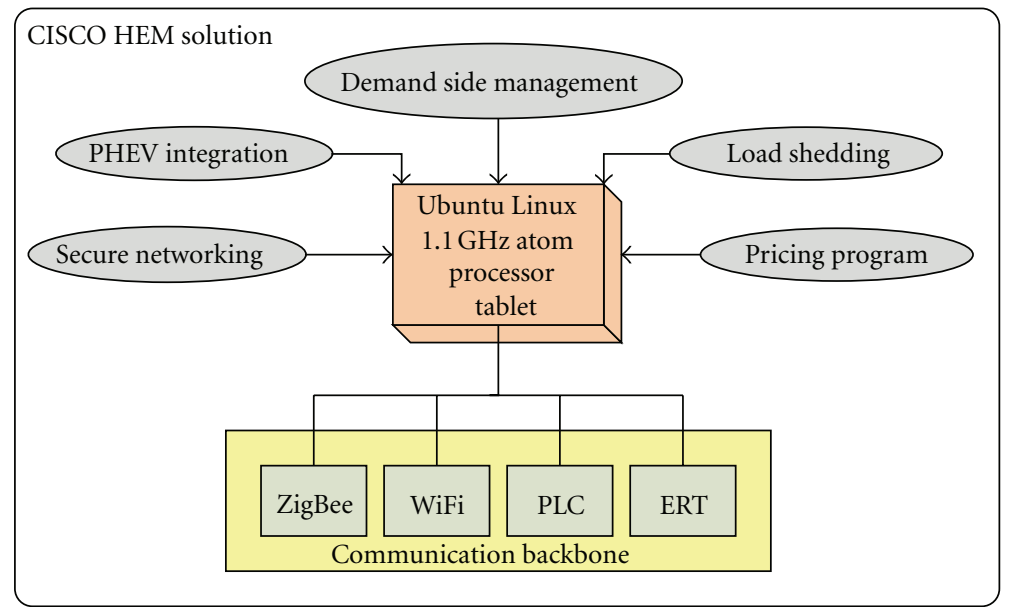

FIgUre 5: Ciscos HEC architecture.

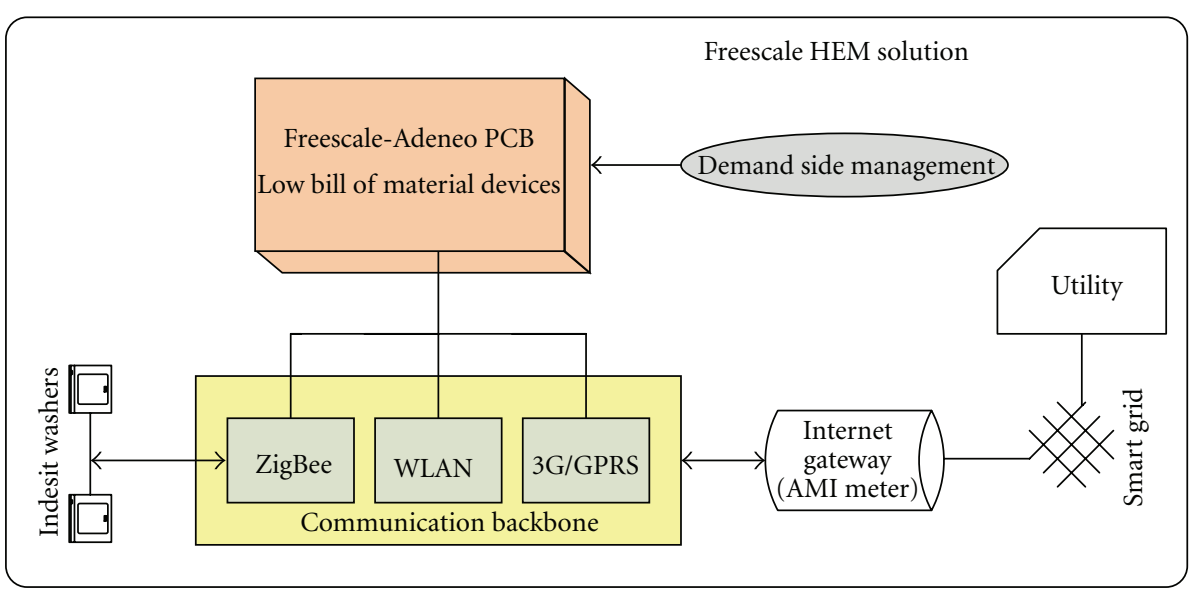

Figure 6: Freescale Technology for a home energy gateway.

Functions of the Home Energy Gateway include

(1) collecting real-time energy consumption from smart meter and power consumption data from various inhouse objects,

(2) controlling activation/deactivation of home appliances,

(3) generating dashboard to provide feedback about power usage,

(4) providing control menus to control appliances,

(5) providing a ubiquitous link to the broadband Internet.

Freescales reference platform is available now through its systems integrator partner Adeneo Embedded, which will provide hardware manufacturing and board support package (BSP) customization and support. The HEG uses a four-layer PCB and boasts a low-cost bill of materials.

In Europe, Freescale announced this summer a smart grid demonstration project with the Indesit Company, an Italian maker of smart appliances. Indesit's Smart Washer was equipped with a Freescale ZigBee node that enables it to adjust its cycle starting time according to energy cost and availability of green power. The washer retrieves this information from the local utility via a ZigBee-enabled Internet connection to the smart grid.

Close on the heels of Freescale, Intel announced its Home Energy Management the acronym HEM reference design earlier in October 2010 Figure 7. Intel's HEM reference design is based on the Atom processor Z6XX series and Intel's Platform Controller Hub MP20. The reference design is manufacturing ready and supports both WiFi and ZigBee. The processor integrates a DDR2 memory controller that can accommodate up to 2 Gbytes of memory.

Intel is marketing the reference design as providing more than just energy management, with the ability to add new applications as they are available. Embedded apps on the dashboard currently include a family message board, weather reports, and home security.

The existing commercial platforms outlined above are the first-generation platforms for HEM. As standardization of control and communication protocols is better adumbrated, and the penetration of HEM use among consumer 


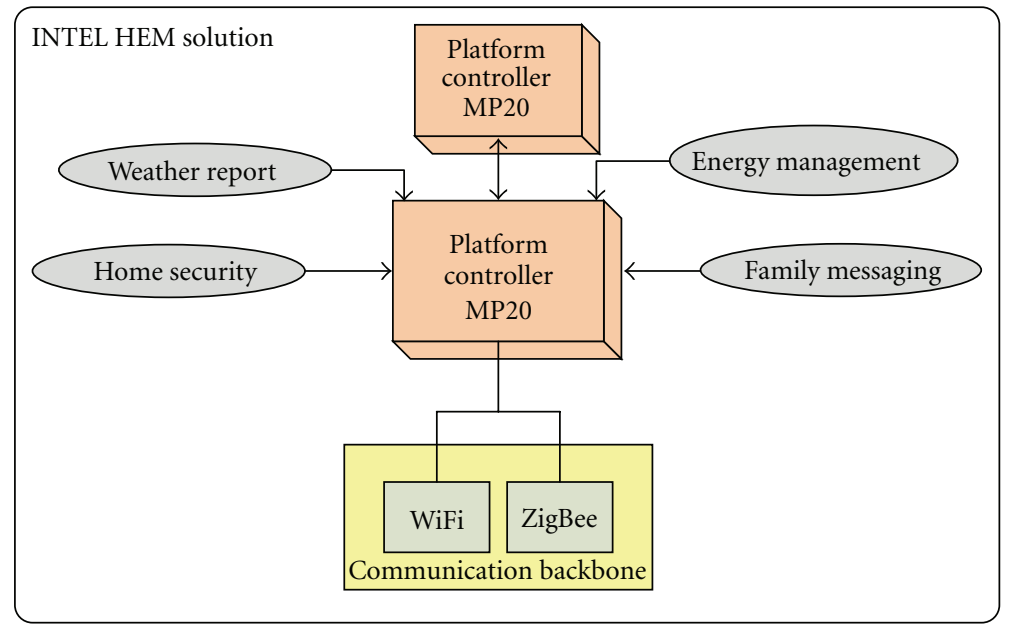

FIGURE 7: Intel HEM solution.

households increases exponentially in the near future, research into designing the optimally efficient and scalable hardware platforms for the next-generation HEM hardware will be paramount. We believe that the next generation HEM devices will also provide various value-added services to the consumers, such as bill payment and security monitoring, for example, besides the expected DSM. Furthermore, these HEM devices will be truly embedded in the HANs, and as is the case with such platforms, the applications and the operating system (OS) which will run on these platforms should be codeveloped and cooptimized with the emerging HEM device architectures.

\section{System Architecture and Challenges in Designing Future HEMs}

In this section, an architecture for a futuristic HEM system is introduced and the challenges and solutions facing the design and deployment of this system are presented. Figure 8 shows an architecture for a future HEM system. Going forward, it is envisioned that a HEM system will be based on an open, nonproprietary, and standards-based platform. This will facilitate the ability to control and network intelligent appliances manufactured by different vendors. The main HEM system can be classified into three subsystems, namely, the Sensor and control devices, the Monitoring and control system, and the Intelligent energy management platform. What follows is a detailed description of these subsystems. It is remarked that while some of these capabilities are available now from a number of HEMS providers, others are future possibilities and will be for quite some time before they hit the market.

5.1. Sensor and Control Devices. This subsystem concerns the basic devices in an HEM system. It is envisioned that future smart home architectures will comprise self-powered (energy scavenging) devices that will facilitate generation of power and energy storage management and diagnostics at a microscale. Other than the power detector it also needs to include the environmental sensor. The main goal is that other than detecting power efficiency it can also detect environmental parameters, such as temperature, humidity, whether there are people active around, and using the HAN set in the environment to send to the intelligent management platform to allow people and other processes to be able to use these information. The controller is used to receive the remote controller commands to control home appliances. The main challenges facing the deployment of an HEM system are summarized below.

(1) Accuracy. For an HEM system, the power detection device should not only give an approximation of the current value, but also accurately measure the current value in the device to enable the intelligent management platform to perform effective appliance recognition function and to determine whether the appliance is operating efficiently using the appliance power source data.

(2) Compatibility. Networking normal home appliances entail integrating the infrared transfer method to the HAN. For example, one can deploy the bridge device discussed in [37] that encodes the received HAN signal into an infrared signal making it compatible with most home appliances. This encoding enables a bidirectional control link between the sensor (on the appliance) and the control device.

(3) Low Power Cost. Detecting the power consumption in a house and the surroundings along with the cost of power consumption requires many strategically deployed detection devices. However, it is essential that these detection devices have low power consumptions and costs and good power management standards, thereby avoiding excessive sensing devices to escalate the cost of power consumption in the HEM system.

5.2. Intelligent Power Management Platform (IPMP). An intelligent power management platform (IPMP) is at the 


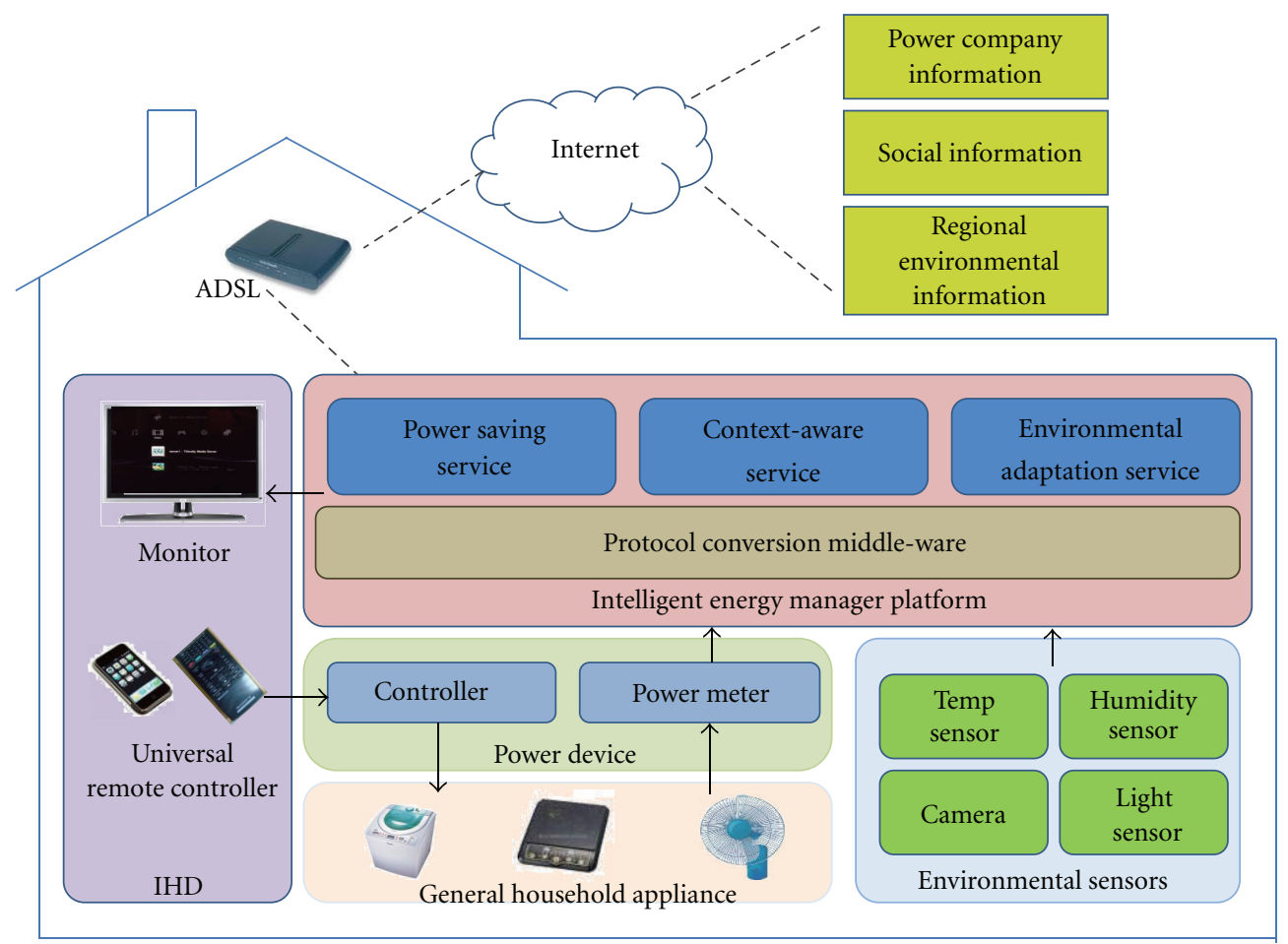

FIgURE 8: Future HEM system architecture.

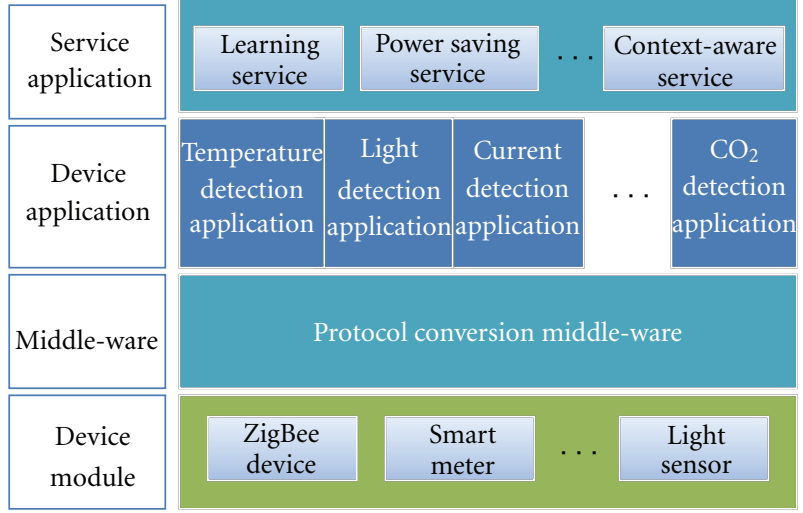

FIGURE 9: Software stack of intelligent power management platform (IPMP).

heart of an HEM system Refer Figure 9. This is because it exploits the received sensor data and external internet data (from power company information, regional environment information, social information, to name a few) and transfers the data to the IHD display for the user. Alternatively, the IPMP automates home control after processing the sensor data in accordance with the "recent" historic sensor data or external information. The IPMP provides a middleware conversion software and allows upper level device and service applications to communicate with each other, thereby facilitating the transfer of data and control signals to lower level devices. The three key services offered by an IPMP are as follows.
(1) Power Management Service. In addition to recording the power usage of each device/appliance, the power management service includes transmitting the power consumption information to the IHD display, and providing appliance recognition and self-managing functions. Through the power sensing device, the power usage of every appliance during the different states of operation is recorded, thereby generating a personalized power consumption profile for each appliance. This power profile can then be used to track and predict the OFF states of an appliance, and this enables a reduction in the power consumed in the whole system by cutting off the power to the appliance during its OFF state. Furthermore, using the power profile of an appliance, one can perform fault analysis to detect a broken or malfunctioning appliance and report it to the user via the IHD.

(2) Context-Aware Service. Context-awareness enables procuring regional environmental information (such as position, climate, and humidity) through the sensor network. This service facilitates recording sensor readings at any time to determine the users' habits, and through further processing and analysis, to automatically control the system under different situations, or using states of the user to prevent wasted power.

(3) Social Network Service. A good intelligent managing platform should also be equipped with a social networking 
function that uses the internet to send the power consumption profiles in a home, and to receive information for the accompanying social networks, including power company data, power costs, and power consumption of each appliance in neighboring homes. Using this information, not only can the user become aware of the power usage in ones' home and, but also the neighborhood using the social network service data to achieve a more detailed power management function. However, there exist security concerns to keep the user information private, and so designing secure and reliable communication links for metering, pricing, control, and billing purposes is areas for future research.

5.3. Monitor and Control System. The main function of this subsystem is to provide a visual interface (such as displaying on the IHD) for the useful information (e.g., power consumption, costs, etc.) for the user to facilitate timely action and control of the HEM system. The design challenge then is to devise a user-friendly and simple integrated control interface for the numerous networked appliances at home. Even though one would envision that universal control panels (i.e., centralized) could offer a good choice for integrating controls, there are still two key challenges.

(1) Integration. Designing an integrated platform that will make the appliances from different vendors operating using different standards interoperable is an open research issue. Using universal controllers entails significant dependence on learning or letting the user record different sets of control signals from different manufacturers to suit each function, thereby limiting convenience and making the deployment of new devices (i.e., scalability of the HAN) harder and expensive.

(2) User Friendliness. Trying to incorporate a number of appliance controls and functions on an single control panel may present result in a panel with numerous control buttons. This might not be the optimal design even for normal users and more so for senior citizens or children. The simplicity and intuitiveness of the user interface will be of paramount importance to the success of smart grids and HEMs in homes. Further, the ease of deployment and upgrading when necessary will preserve the customer base for smart home technologies.

\section{Conclusions}

On a concluding note, the need for smart energy management in the residential sector for sustainable energy efficiency and monetary savings was revisited in this paper. As the smart grid extends out to homes and businesses, wireless sensors and mobile control devices become important elements in monitoring and managing energy use. There are several challenges of which smart energy system designers need to be aware. One challenge is the fragmentation of the HAN market. There are several wireless standards that are currently used in HANs including WiFi, ZigBee, Z-Wave, and Bluetooth; however, despite the emergence of many wireless standards for HANs, there is no clear winner at this point, and so it is up to the system designers to select a wireless technology that best fits their application while addressing the potential problem of interoperability with other HAN devices. A comprehensive summary of the state of the art in home area communications and networking technologies for energy management was provided in the paper, followed by a review of the affordable smart energy products offered by different companies. The paper also shed light on the challenges facing the design of future energy management systems, such as the need for interoperability and network security. Our discussions will hopefully inspire future efforts to develop standardized and more user-friendly smart energy monitoring systems that are suitable for widescale deployment in homes.

\section{References}

[1] G. T. Gardner and P. C. Stern, "The most effective actions U.S. households can take to curb climate change," Environment, vol. 50 , no. 5 , pp. $12-25,2008$.

[2] http://wiki.micasaverde.com/index.php/Energy_Savings.

[3] M. Fitzgerald, "Finding and fixing a homes power hogs," New York Times, 2008, http://www.nytimes.com/2008/07/27/technology/27proto.html.

[4] C. W. Gellings, The Smart Grid: Enabling Energy Efficiency and Demand Response, CRC Press, 2009.

[5] D. J. Cook and S. K. Das, "How smart are our environments? An updated look at the state of the art," Pervasive and Mobile Computing, vol. 3, no. 2, pp. 53-73, 2007.

[6] K. Kok, S. Karnouskos, D. Nestle et al., "Smart houses for a smart grid," in the 20th International Conference and Exhibition on Electricity Distribution (CIRED '09), June 2009.

[7] J. R. Roncero, "Integration is key to smart grid management," in IET-CIRED Seminar on SmartGrids for Distribution, vol. 2008, June 2008.

[8] M. Erol-Kantarci and H. T. Mouftah, "Wireless sensor networks for domestic energy management in smart grids," in 25th Queen's Biennial Symposium on Communications (QBSC '10), pp. 63-66, Kingston, Canada, May 2010.

[9] D. G. Hart, "Using AMI to realize the Smart Grid," in IEEE Power and Energy Society General Meeting: Conversion and Delivery of Electrical Energy in the 21st Century, PES, pp. 1-2, July 2008.

[10] C. Hertzog, Smart Grid Dictionary, GreenSpring Marketing, 1st edition, 2009.

[11] H. Farhangi, "The path of the smart grid," IEEE Power and Energy Magazine, vol. 8, no. 1, pp. 18-28, 2010.

[12] K. Wacks, "The gridwise vision for a smart grid," Parks Associates' CONNECTIONS, June 2009.

[13] M. Inoue, T. Higuma, Y. Ito, N. Kushiro, and H. Kubota, "Network architecture for home energy management," IEEE Transactions on Consumer Electronics, vol. 49, no. 3, pp. 606613, 2003.

[14] N. Kushiro, S. Suzuki, M. Nakata, H. Takahara, and M. Inoue, "Integrated residential gateway controller for home energy management system," IEEE Transactions on Consumer Electronics, vol. 49, no. 3, pp. 629-636, 2003.

[15] R. Masiello, "Demand Response: the other side of the curve," IEEE Power and Energy Magazine, vol. 8, no. 3, p. 18, 2010. 
[16] US Department of Energy, "Benefits of Demand Response in Electricity Markets and Recommendations for Achieving Them".

[17] M. H. Albadi and E. F. El-Saadany, "Demand response in electricity markets: an overview," in IEEE Power Engineering Society General Meeting (PES '07), June 2007.

[18] Charles River Associates, "Primer on demand-side management With an emphasis on price-responsive programs," Prepared for The World Bank by Charles River Associates, February 2005.

[19] EPRI, "Survey of utility demand-side management programs," EPRI TR-102193, 1993.

[20] NERC, Electricity Supply and Demand, for 1993-2002, North American Electric Reliability Council, Princeton, NJ, USA, 1993.

[21] Barakat \& Chamberlin, Inc, Principles and Practice of DemandSide Management, EPRI TR-102556, Palo Alto, Calif, USA, 1993.

[22] A. Brooks, E. Lu, D. Reicher, C. Spirakis, and B. Weihl, "Demand dispatch," IEEE Power and Energy Magazine, vol. 8, no. 3, pp. 20-29, 2010.

[23] A. Vojdani, "Smart integration," IEEE Power and Energy Magazine, vol. 6, no. 6, pp. 71-79, 2008.

[24] N. Gudi, L. Wang, V. Devabhaktuni, and S. S. S. R. Depuru, "Demand response simulation implementing heuristic optimization for home energy management," in the North American Power Symposium (NAPS '10), September 2010.

[25] T. C. Matty, "Advanced energy management for home use," IEEE Transactions on Consumer Electronics, vol. 35, no. 3, pp. 584-588, 1989.

[26] T. Ikegami, Y. Iwafune, and K. Ogimoto, "Optimum operation scheduling model of domestic electric appliances for balancing power supply and demand," in the International Conference on Power System Technology (POWERCON '10), October 2010.

[27] T. Verschueren, W. Haerick, K. Mets, C. Develder, F. De Turck, and T. Pollet, "Architectures for smart end-user services in the power grid," in the IEEE/IFIP Network Operations and Management Symposium Workshops (NOMS '10), pp. 316322, April 2010.

[28] D. L. Ha, F. F. De Lamotte, and Q. H. Huynh, "Real-time dynamic multilevel optimization for Demand-side Load management," in the IEEE International Conference on Industrial Engineering and Engineering Management (IEEM '07), pp. 945-949, December 2007.

[29] K. P. Wacks, "Utility load management using home automation," IEEE Transactions on Consumer Electronics, vol. 37, no. 2, pp. 168-174, 1991.

[30] M. H. Shwehdi and A. Z. Khan, "A power line data communication interface using spread spectrum technology in home automation," IEEE Transactions on Power Delivery, vol. 11, no. 3, pp. 1232-1237, 1996.

[31] L. D. Ha, S. Ploix, E. Zamai, and M. Jacomino, "Tabu search for the optimization of household energy consumption," in IEEE International Conference on Information Reuse and Integration (IRI '06), pp. 86-92, September 2006.

[32] S. Kishore and L. V. Snyder, "Control mechanisms for residential electricity demand in smartgrids," in Proceedings of the 1st IEEE International Conference on Smart Grid Communications (SmartGridComm '10), pp. 443-448, 2010.

[33] B. Ablondi, "Residential energy management," Parks Associates' CONNECTIONS, June 2009.

[34] Z-Wave Alliance, http://www.Z-wavealliance.org/modules/AllianceStart/.
[35] U. Saif, D. Gordon, and D. J. Greaves, "Internet access to a home area network," IEEE Internet Computing, vol. 5, no. 1, pp. 54-63, 2001.

[36] E. Callaway, P. Gorday, L. Hester et al., "Home networking with IEEE 802.15.4: a developing standard for low-rate wireless personal area networks," IEEE Communications Magazine, vol. 40, no. 8, pp. 70-77, 2002.

[37] W. K. Park, I. Han, and K. R. Park, "ZigBee based dynamic control scheme for multiple legacy IR controllable digital consumer devices," IEEE Transactions on Consumer Electronics, vol. 53, no. 1, pp. 172-177, 2007. 

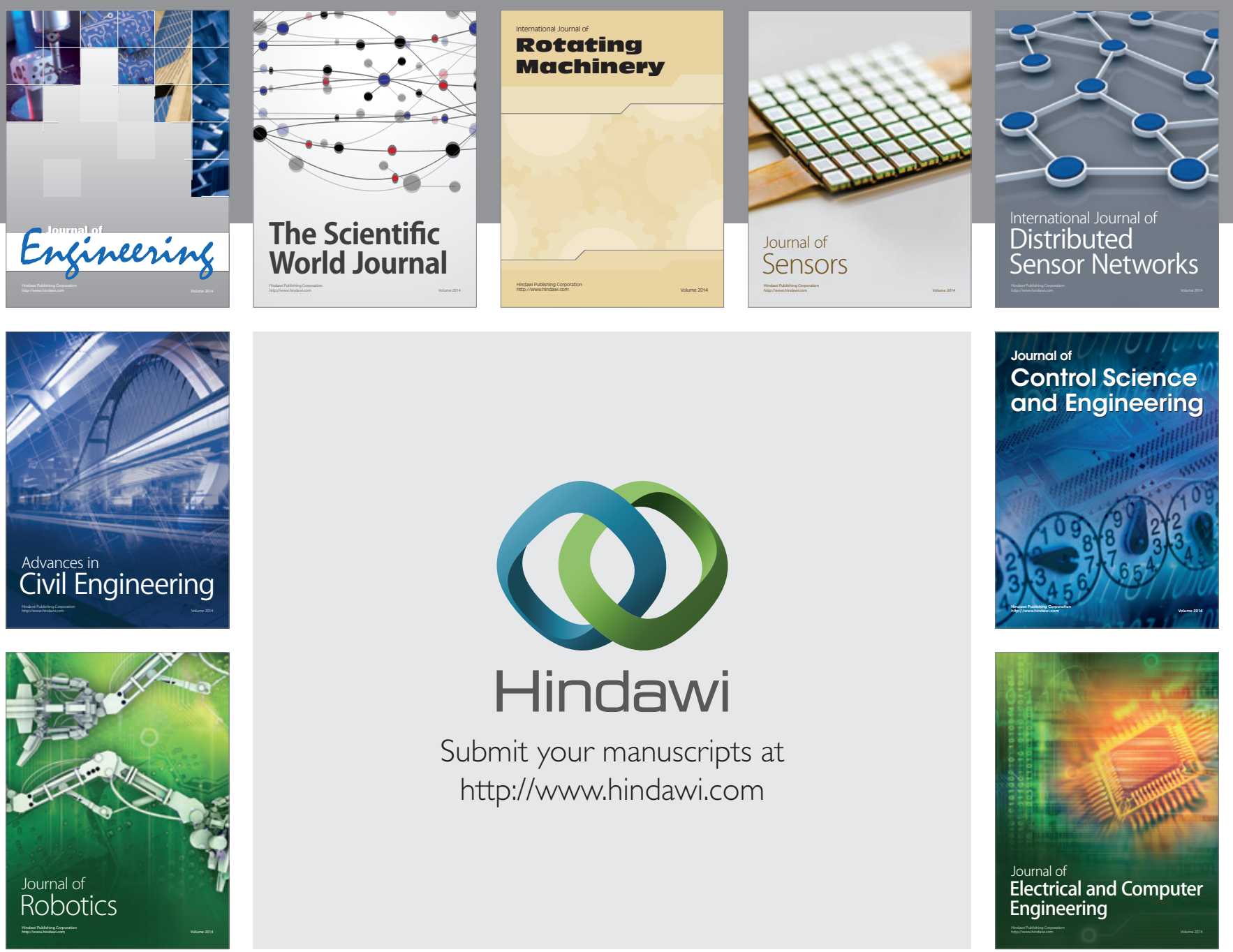

Submit your manuscripts at

http://www.hindawi.com
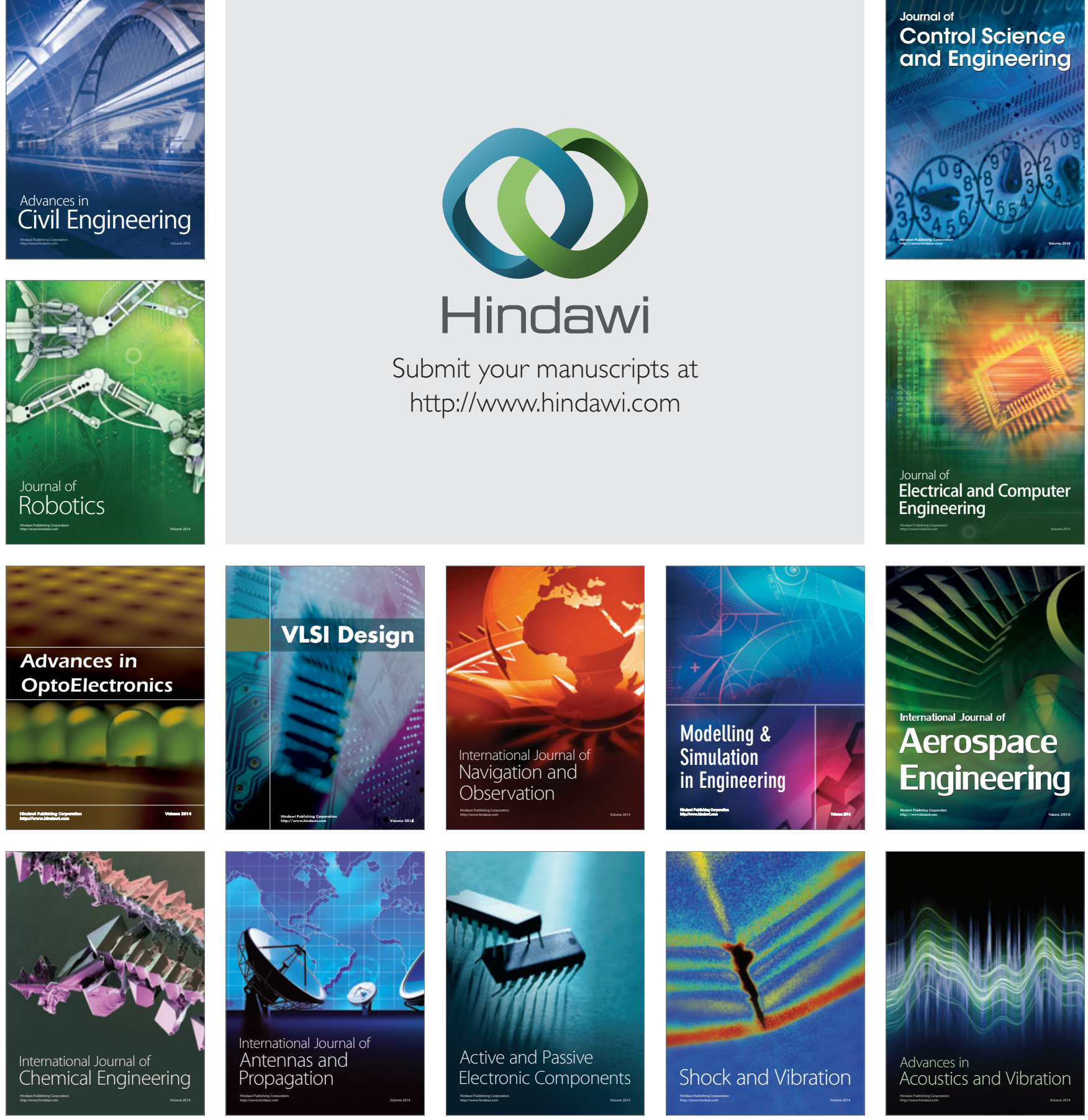